\title{
Evaluation of Fatigue Resistance of Acetal Resin and Cobalt-Chromium Removable Partial Denture Clasps. An In-vitro Study: Part 1
}

\author{
Abd-Elrahman IA ${ }^{1}$, Helal $\mathrm{MA}^{{ }^{2}}$, Saqar $\mathrm{HM}^{3}$ and $\mathrm{Abas}^{4}$ \\ ${ }^{1}$ Dentist at the MOH, El-Mansoura, Egypt \\ ${ }^{2}$ Professor of Removable Prosthodontics, Faculty of Dental Medicine, Al-Azhar University, Boys Branch, Cairo, \\ Egypt \\ ${ }^{3}$ Assistant Professor of Removable Prosthodontics, Faculty of Dental Medicine, Al-Azhar University, Boys Branch, \\ Cairo, Egypt \\ ${ }^{4}$ Assistant Professor of Dental Material, Faculty of Dental Medicine, Al-Azhar University, Boys Branch, Cairo, Egypt \\ ${ }^{*}$ Corresponding author: Helal MA, Professor of Removable Prosthodontics, Faculty of Dental Medicine, Al- \\ Azhar University, Boys Branch, Cairo, Egypt, E-mail: mhelal71@gmail.com
}

Citation: Abd-Elrahman IA, Helal MA, Saqar HM, Abas M (2016) Evaluation of Fatigue Resistance of Acetal Resin and Cobalt-Chromium Removable Partial Denture Clasps. An In-vitro Study: Part 1. J Dent Oral Care Med 2(3): 304. doi: 10.15744/2454-3276.2.304

Received Date: August 02, 2016 Accepted Date: August 30, 2016 Published Date: August 31, 2016

\begin{abstract}
Purpose: This study was aimed to evaluate the retentive forces of acetal resin clasps and Cobalt-Chrome (Co-Cr) clasps during attachment/detachment cycles on abutment teeth with two different undercuts and at different intervals.

Materials and Method: Twenty models were constructed by placing either an upper $1^{\text {st }}$ premolar or upper $1^{\text {st }}$ molar inside an acrylic rectangular block. Models were divided according to the abutment teeth into two groups (GI for $1^{\text {st }}$ premolars group and GII for $1^{\text {st }}$ molars group), 10 each. Each group was divided into two subgroups according the framework material, SGA for acetal resin clasp and SGC for Co-Cr clasp. Each testing models and its framework were mounted inside universal testing machine (Lloyd instruments Ltd, England). Retention of each clasp at pre-test ( 0 cycle) and after cycling intervals ( 730 cycles, 1460 cycles, 2190 cycles, and 2920 cycles) were measured by applying withdrawal force to it using universal testing machine. The data of the retentive force magnitudes at different intervals were collected and tabulated. The data were subjected to statistical analysis using 3-way-ANOVA test, student's $t$ and paired $t$ tests.

Results: After 730 cycles, 1460 cycles, 2190 cycles, and 2920 cycles there were non significant difference between SGIA and SGIC, and SGIA and SGIIA at $95 \%$ confidence level $(\mathrm{P}>0.05)$, however there were statistical significant difference between SGIIA and SGIIC, and SGIC and SGIIC at $95 \%$ confidence level $(\mathrm{P} \leq 0.05)$.

Conclusion: Acetal resin clasps ( $1.0 \mathrm{~mm}$ in cross section diameter) engage $0.25 \mathrm{~mm}$ provide sufficient retention nearly similar that of Co-Cr clasps, The acetal resin clasps should be thicker more than $1.0 \mathrm{~mm}$ (in cross-sectional diameter) when engage a deeper undercut $(0.50 \mathrm{~mm})$ to gain the stiffness of the cast Co-Cr clasps $1 \mathrm{~mm}$ in cross sectional diameter and to obtain clinically acceptable retention.
\end{abstract}

Keywords: Clasp; Acetal Resin; Cobalt-Chrome; Deflection Fatigue

\section{Introduction}

Sufficient retention and esthetics of removable partial dentures (RPDs) are considered as the most important factors affecting their clinical success. So the achieving of optimal esthetics while maintaining retentive integrity, and preserving the health of the abutment is a big dilemma [1-3].

The most common metal alloy used for construction of the direct retainers of RPDs is a cobalt-chromium (Co-Cr) alloy, also other alloys as gold, titanium alloy and round wrought wire can be used for fabrication of the direct retainers [4-14].

The display of the direct retainer assemblies of RPD is a big esthetical problem [12]. Many trials have been carried out to overcome this problem such as etching the clasp arm and coating it with a layer of tooth-color resin, and lingual retention design [13,15-17].

Recently thermoplastic resins have been introduced into the market, direct retainers fabricated in a tooth-colored material and constructed from polyoxymethylene (POM), also the polyetheretherketon (PEEK), and polyetherketonketon (PEKK) have been used to enhance the esthetic of metal direct retainer assemblies of RPD $[17,18]$. 
POM is highly versatile engineering polymer that bridges the gap between metal and ordinary plastic, because it offer the strength of metal and the flexibility and comfort of plastic [3]. Also it is called by acetal resin, and it has been used as a alternative for acrylic resins in many dental prosthetic situations [19,20].

The design, retentive force, colour stability, water sorption and solubility of acetal resin clasps have been investigated [20-23]. While, Ozkan et al. showed clinically acceptable discolouration values for polyacetal resin that subjected to thermocycling [24].

Retentive clasp arms of the direct retainers must be flexible and should retain the RPD satisfactorily without not unduly stress abutment teeth or be permanently deformed during service. Direct retainers of the RPD that were constructed from elastic materials demonstrated a higher resistance to retention loss $[25,26]$.

In spite of the thermoplastic resin direct retainer is more flexible than the conventional Co-Cr direct retainer there is little data available regarding the long-term performance of such direct retainers [19,27-31]. Therefore this study was aimed to evaluate the retentive forces of acetal resin clasps and Co-Cr clasps during attachment/detachment cycles on abutment teeth with two different undercuts and at different intervals. The null hypothesis of this study was that the differences in the retentive forces between resin clasps and Co-Cr alloy clasps at different intervals and two amounts of undercut $(0.25 \mathrm{~mm}$ and $0.50 \mathrm{~mm})$ would be insignificant.

\section{Materials and Methods}

For this study twenty natural teeth (ten maxillary $1^{\text {st }}$ premolars and ten maxillary ${ }^{\text {st }}$ molar) were used for construction twenty testing models to create an apparatus to hold the abutment during testing as following:

One laboratory custom made copper model (30 mm in length, $20 \mathrm{~mm}$ in width, and $25 \mathrm{~mm}$ in height) was used for fabrication the testing models. The testing models were constructed from rectangular acrylic (Stellon, DeguDent Gmbh, and England) blocks with a natural tooth embedded in each model vertically to the cementoenamel junction.

The testing models were divided into two groups according to the model's teeth: group I (GI) contained ten testing models, each testing model having $1^{\text {st }}$ premolar $(0.25 \mathrm{~mm}$ undercut), and group II (GII) contained ten testing models, each testing model having $1^{\text {st }}$ molar (0.50 mm undercut).

The abutment of the testing model was surveyed to ensure that there was adequate undercut ( $0.25 \mathrm{~mm}$ for GI and 0.50 for GII) [14]. Minor tooth preparations were performed to provide rest seat. The palatal surface was recontoured by using stone with low speed hand piece for lowering the height of contour to provide ideal balance (reciprocation) between two arms of the clasp [1,2].

Then a small piece of wax $(0.7 \mathrm{~mm}$ in thickness, $20 \mathrm{~mm}$ in length, and $5 \mathrm{~mm}$ in width) having a small perforation at the its distal end, was fixed at the superior surface of the testing model, and $2 \mathrm{~mm}$ from the proximal tooth surface to provide framework stopper. The unwanted proximal undercuts were blocked out by using softened wax to eliminate its effect on the retention force and then trimmed by means of the wax trimmer of dental surveyor $[1,2,11]$.

The testing models were duplicated into investment models (Calibra-M, Protechno, Spain) by using silicone duplicating material (Dupliflex, Protechno, Germany).

According the clasp and framework material each group was divided into two subgroup (SG), each SG contained five testing models, SGA for acetal resin clasp material and SGC for Cr-Co clasp material.

On the investment models, cast Co-Cr alloy (Kera C, Eisenbacher Dentalwaren ED GmbH, and Germany) and acetal resin material (Bredent, Germany) frameworks with Aker clasps were constructed as following.

Half round cross section Aker clasp wax patterns with $1.0 \mathrm{~mm}$ thickness (Polywax, Bilkim, and Izmir, Turkey) were used to construct the wax patterns of all frameworks of this study. After that a small rectangular piece of wax (15 mm in length, $3 \mathrm{~mm}$ in height, and $6 \mathrm{~mm}$ in width) was fixed on the superior surface of the investment cast with $2 \mathrm{~mm}$ away from the distal surface of the abutment, by using softened wax. This rectangular plastic piece was placed parallel to superior surface of the investment cast; it was connected with the wax pattern of the clasp by wax. A small cylindrical piece of wax sprue ( $20 \mathrm{~mm}$ in length and $3 \mathrm{~mm}$ in diameter) was fixed at 90 degree to the rectangular piece of wax (to produce a testing column of the framework) and away 3 mm from the minor connector of the wax pattern framework.

The flasking and the injection process of acetal resin for the wax pattern of the frameworks of SGIA and SGIIA were carried out using the acetal furnace (Thermopress 400, Bredent, Seden, Germany), however the wax pattern of frameworks of SGIC and SGIIC were cast into Co-Cr as conventional manner, (Figure 1a-1c).

The frameworks were tried on the models and were considered to be suitable for testing when the occlusal rests fit well in their rest seats, the retainers were in contact with the abutments, and the positive part of the framework rested on the testing model.

Heavy contacts of the Co-Cr and the acetal resin minor connectors with the proximal surfaces of the tooth were adjusted using the disclosing medium (Impregum Soft, 3M ESPE, and USA). The disclosing medium was applied, the framework was gently inserted into the testing model and held firmly until the silicone has set [1,2]. The framework was removed and carefully checked. Areas 
that were exhibited metal showing through were adjusted. Then old disclosing medium was cleaned off, and new medium was applied, and the framework was tried in again until seating was achieved.

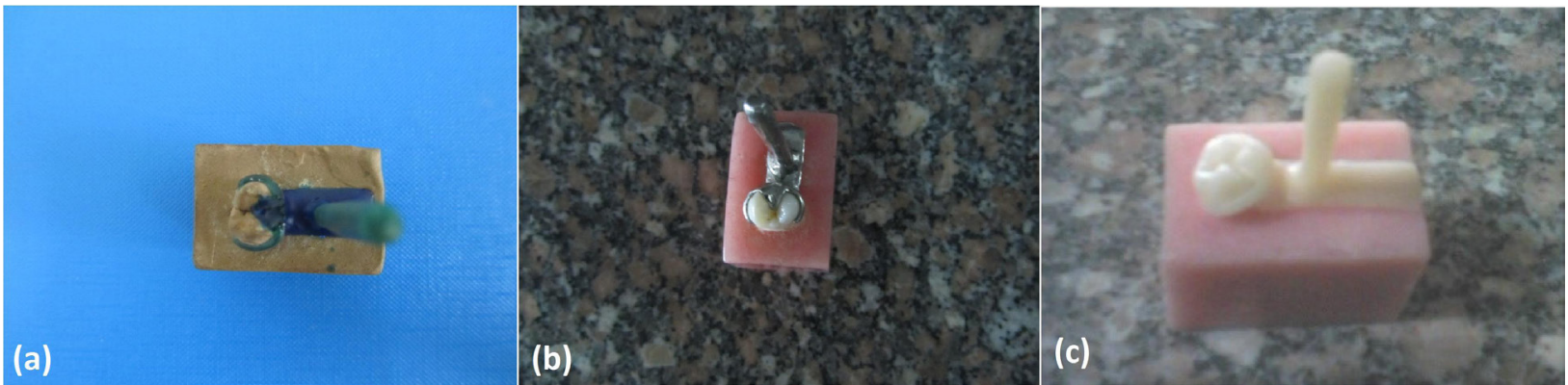

Figure 1: (a) Wax pattern of Aker clasp, (b) Co-Cr Aker clasp, and (c) Acetal resin Aker clasp.

Each clasp and its model were mounted on a universal testing machine (Lloyd instruments Ltd, England). Retention of each clasp at pre-test ( 0 cycles) was measured by applying withdrawal force to it by this machine. Removal and insertion cycling of clasps was carried out for 730, 1460, 2190, 2920 cycles (corresponding to 6, 12, 18 and 24 months of simulated clinical use of a RPD) to simulate the fatigue resistance test [1,2]. Specimens were cycled at room temperature. The machine was set at $0.5 \mathrm{~mm} / \mathrm{min}$ with 3 $\mathrm{kg}$ loading. The retention force was considered as the maximum load that required removing the clasp at 0, 730, 1460, 2190, and 2920 cycles, and it was recorded by the computer software (Nexygen-MT; Lloyd instruments).

The data of the retentive force magnitudes in $\mathrm{N}$ at different intervals were collected and tabulated. The data were subjected to statistical analysis using 3-way-ANOVA test, 2-way-ANOVA test, student's t test and paired t test.

\section{Results}

Table 1 shows the means and standard deviations of retention forces of different subgroubs at different intervals. However, Table 2 shows the pairwise compariosns of the retention forces of different subgroups at different intervals.

At pretest (0 cycling), pair-wise comparisons using student's t test showed non significant difference between SGIA and SGIC at 95\% confidence level (P > 0.05), and showed statistical significant difference between SGIIA and SGIIC at 95\% confidence level (P $\leq 0.05)$. While paired t test showed statistically non-significant between SGIA and SGIIA, and SGIC and SGIIC at $95 \%$ confidence level $(\mathrm{P}>0.05)$.

After each interval (730 cycles, 1460 cycles, 2190 cycles, and 2920 cycles) student's t test showed non significant difference between SGIA and SGIC at 95\% confidence level (P > 0.05), and there was statistical significant difference between SGIIA and SGIIC at 95\% confidence level $(\mathrm{P} \leq 0.05)$. There was statistical non siginficant differennce between SGIA and SGIIA at 95\% confidence level $(\mathrm{P}>0.05)$, and there was statistical significant difference between SGIC and SGIIC using Paired test, at 95\% confidence level (P $\leq 0.05$ ).

At the base line, regardless to abutment undercuts 2-way-ANOVA test showed statistically significant difference between Cr-Co clasps subgroups and acetal resin clasps subgroups $(\mathrm{P} \leq 0.05)$. Irrespective of clasp material the difference between molar group and premolar group was statistically non-significant $(\mathrm{P}>0.05)$. After different cycling intervals, regardless to abutment undercuts 2-way-ANOVA test showed statistically significant difference between Cr-Co clasps subgroups and acetal resin clasps subgroups $(\mathrm{P} \leq 0.05)$. Also irrespective of clasp material the difference between molar group and premolar group was statistically significant $(\mathrm{P} \leq 0.05)$.

Statistically, 3-way-ANOVA test showed statistically significant effect of clasp material, effect of amount of abutment undercut, and mechanical cycling (aging) on the retention force, at 95\% confidence level $(\mathrm{P} \leq 0.05)$.

\begin{tabular}{|c|c|c|c|c|c|}
\hline Subgroups & \multicolumn{5}{|c|}{ Mean \pm SD } \\
\hline & 0 Cycle & $\mathbf{7 3 0}$ Cycles & $\mathbf{1 4 6 0}$ Cycles & $\mathbf{2 1 9 0}$ Cycles & 2920 Cycles \\
\hline SGIA & $19.195 \pm 0.34$ & $9.48 \pm 2.5$ & $4.27 \pm 1.5$ & $2.39 \pm 0.8$ & $1.46 \pm 0.3$ \\
\hline SGIC & $21.982 \pm 3.8$ & $8.8 \pm 1.9$ & $2.01 \pm 0.74$ & $1.92 \pm 0.48$ & $1.58 \pm 0.49$ \\
\hline SGIIA & $19.16 \pm 0.67$ & $8.25 \pm 0.77$ & $3.13 \pm 0.42$ & $2.48 \pm 0.39$ & $1.88 \pm 0.68$ \\
\hline SGIIC & $25.025 \pm 1.1$ & $19.67 \pm 1.8$ & $12.2 \pm 2.6$ & $7.25 \pm 2.8$ & $5.14 \pm 2.8$ \\
\hline
\end{tabular}

Table 1: Means and standard deviations of retention forces of different subgroubs at different intervals

SGIA, Subgroub of Aker acetal clasp that engaed $0.25 \mathrm{~mm}$ undercut. SGIC, Subgroub of Aker Cr-Co clasp that engaed $0.25 \mathrm{~mm}$ undercut. SGIIA, Subgroub of Aker acetal clasp that engaed $0.50 \mathrm{~mm}$ undercut. SGIIC, Subgroub of Aker Cr-Co clasp that engaed $0.25 \mathrm{~mm}$ undercut. 


\begin{tabular}{|c|c|c|c|c|c|c|c|c|c|c|}
\hline \multirow{2}{*}{$\begin{array}{c}\text { Pairwise } \\
\text { Comparisons }\end{array}$} & \multicolumn{2}{|c|}{0 Cycle } & \multicolumn{2}{|c|}{730 Cycles } & \multicolumn{2}{|c|}{1460 Cycles } & \multicolumn{2}{|c|}{2190 Cycles } & \multicolumn{2}{|c|}{2920 Cycles } \\
\hline & $t$ & $P$ & $t$ & $P$ & $t$ & $P$ & $t$ & $P$ & $t$ & $P$ \\
\hline SGIA Vs SGIC & 1.6121 & 0.1456 & 1.499 & 0.1720 & 0.7817 & 0.4568 & 2.1397 & 0.0648 & 0.2192 & 0.8319 \\
\hline SGIA Vs SGIIA & 0.1584 & 0.8818 & 0.7983 & 0.4693 & 0.2126 & 0.8420 & 2.1241 & 0.1008 & 3.1024 & 0.0361 \\
\hline SGIIA Vs SGIIC & 10.244 & $<0.0001^{*}$ & 7.7589 & $<0.0001^{*}$ & 3.7978 & $0.00525^{\star}$ & 4.6072 & $0.0017^{\star}$ & 5.3375 & $0.00069^{*}$ \\
\hline SGIC Vs SGIIC & 2.326 & 0.0805 & 8.274 & $0.0011^{\star}$ & 6.7930 & $0.00245^{\star}$ & 5.5686 & $0.0050^{*}$ & 5.9835 & $0.0039^{*}$ \\
\hline
\end{tabular}

* denotes significant differenece $(\mathrm{P} \leq 0.05)$

Table 2: Pairwise compariosns of the retention forces of different subgroups at different intervals

\section{Discussion}

An in-vitro study was carried out to compare the retention forces of acetal resin clasps and Co-Cr clasps with two amounts of undercut at different intervals. This experiment was conducted for 2920 cycles to simulate approximately an two years period, if an RPD would be removed four times each day for two years [1,2].

The demand for esthetic dental restorations has been increased due to emphasis on physical appearance in contemporary society [30]. Therefore the $0.25-\mathrm{mm}$ undercut was selected because it represents the undercut that commonly used for Co-Cr clasps. With the increase the need for esthetics, the patients are asking the prosthodntists to conceal RPD direct retainers by placing them more cervically, however the undercuts of this area tend to be larger. So the second undercut condition $(0.50 \mathrm{~mm})$ was chosen to determine whether construction of RPD direct retainers from acetal resin would be a possible substitute to the metal clasps in large undercuts [21].

Loss of retention of the clasps due to fatigue resistance test was considered as a good indicator of permanent deformation of the clasps $[6,25]$.

Initially, the mean of retention force in N was 19.195 for SGIA, 21.982 for SGIC, 19.16 for SGIIA, and 25.025 for SGIIC. The pervious results were higher than that recorded with other studies and were disagreement with Helal, et al. Firtell and Soo S, Leung $[1,2,9,11]$. The variations in retention forces compared with the previous studies may be due to the difference in the flexibility of the alloys used, or the amount of deflection, or the testing models.

The difference in the alloys used lead difference in the flexibility that will affect the retention; the difference in the amount of the abutment undercuts leads to difference in the amount of deflection that will affect the retention. Also the difference in the design of the testing models affect the retention due to the different of the direction of the insertion and withdrawal forces that will affect the retention.

After 2920 cycles the mean of retention force in N was 1.46 for SGIA, 1.58for SGIC, 1.88 for SGIIA, and 5.14 for SGIIC, these were disagreement with other studeies. However Tannous, et al. stated that "the mean retentive force for the $1.0 \mathrm{~mm}$ thick thermoplastic resin clasps at the end of the cycling test ranged from $1.7 \mathrm{~N}$ to $3.7 \mathrm{~N}$, and for the $1.5 \mathrm{~mm}$ thick clasps from $5.4 \mathrm{~N}$ to $10.8 \mathrm{~N}$ " $[1,2,14]$.

After 730 cycles, 1460 cycles, 2190 cycles, and 2920 cycles there were no significant differences between SGIA and SGIC, and SGIA and SGIIA at 95\% confidence level ( $\mathrm{P}>0.05$ ), however, there were statistical significant differences between SGIIA and SGIIC, and SGIC and SGIIC at 95\% confidence level $(\mathrm{P} \leq 0.05)$, these may be attributed to the effect for the clasp material, and amount of abutment undercut on the retention forces. Therefore the null hypothesis of this study that there would be no significant difference in the retention forecs of acetal resin clasps and Co-Cr clasps at different intervals and two amounts of undercut $(0.25 \mathrm{~mm}$ and $0.50 \mathrm{~mm}$ ) was rejected.

The results of the current study revealed that there were significant effect for the clasp material, amount of abutment undercut, and mechanical cycling (aging) on the retention forces at different intervals $(\mathrm{P} \leq 0.05)$.

There was a significant effect for the clasp material on the retention forces of the clasps at different intervals $(\mathrm{P} \leq 0.05)$. The results of this study revealed that there were significant increase in retention forces for the $\mathrm{Cr}$-Co clasps that engaged $0.50 \mathrm{~mm}$ undercut more than that of acetal clasps at different intervals $(\mathrm{P} \leq 0.05)$, these were in agreement with Tannous, et al. and Arda and Arikan who reported that the mean of retention force of Co-Cr clasps was significantly higher than that for acetal resin clasps [14,21]. However, there were non significant differences in retention forces between acetal resin clasps that engaged $0.25 \mathrm{~mm}$ undercut and that of Co-Cr clasps at different intervals $(\mathrm{P}>0.05)$. Moreover, there was no significant difference in retention forces between acetal resin clasps that engaged $0.25 \mathrm{~mm}$ undercuts and acetal resin clasps that engaged $0.50 \mathrm{~mm}$ undercuts however there were significant differences in retention forces between Co-Cr clasps that engaged $0.25 \mathrm{~mm}$ undercuts and Co-Cr clasps that engaged $0.50 \mathrm{~mm}$ undercuts.

This may be due to the modulus of elasticity for acetal resin is $2.36 \mathrm{GPa}$, that of Co-Cr alloy is $240 \mathrm{GPa}$, the relatively low rigidity of the acetal resin, and similarity in the thickness of acetal resin clasps and Co-Cr clasp that used in this study. Therefore the acetal resin clasps should be thicker (in cross-sectional diameter) than Co-Cr clasps when engages a deeper undercut $(0.50 \mathrm{~mm})$ to 
gain clinically acceptable retention. However, the increase thickness (in cross-sectional diameter) of the acetal clasp may lead to plaque stagnation, so RPD with proper design might not cause such problem if the plaque control is carried out and reinstruction is provided $[12,13]$.

Also there were significant increase in retention force for Co-Cr clasps of molar group ( $0.50 \mathrm{~mm}$ undercut $)$ more than that of CoCr clasps of premolar group $(0.25 \mathrm{~mm}$ undercut $)(\mathrm{P} \leq 0.05)$ due to increase the amount of the undercut. These were in agreement with Tannous, et al. who mentioned that "the clasps with $0.25 \mathrm{~mm}$ undercut showed significantly less retention than clasps with $0.50 \mathrm{~mm}$," and with Arda and Arikan who reported that "the retentive force needed to dislodge clasps was significantly lower for the models with $0.25 \mathrm{~mm}$ undercuts than for those with $0.50 \mathrm{~mm}$ undercuts" [14,21].

There was significant effect for mechanical cycling (aging) on the retention forces at different intervals $(\mathrm{P} \leq 0.05)$. There was gradual loss of the retention forces of all clasps of this study after different cycling intervals. These were in accord with Helal, et al. who reported that the clasp may lose its retention force as a result of multiple deflections, and at variance with Meenakshi, et al. who showed that the retention force of acetal clasps did not decrease after cycling intervals while the Co-Cr clasps lost retention force within 730 cycles of insertion/removal and continued to lose retention force during the remaining intervals [1,2,31].

Finally, the results of the present study were in accord with Fitton, et al. who stated that "the POM clasps must have greater crosssection area than metal clasps to provide adequate retention," in accordance with these results, Tannous, et al. reported that the greatest retentive force for acetal clasps was found in the $1.5 \mathrm{~mm}$ thick clasps designed to engage the $0.50 \mathrm{~mm}$ undercut [14,19]. Also others mentioned that the POM clasp must be greater in cross-sectional diameter (approximately $1.4 \mathrm{~mm}$ ) and approximately $5 \mathrm{~mm}$ shorter than Co-Cr clasps in order to have the stiffness similar to a cast Co-Cr clasp $1 \mathrm{~mm}$ in cross-sectional diameter and $15 \mathrm{~mm}$ long [20]. On the other hand, these results were disagreeing with others who reported that the proportional limit of acetal resin enabling it to engage large undercut [21,27]. Also others reported that acetal resin has superior flexibility compared to the Co-Cr alloys $[13,17,18]$.

This study suggests investigating the fatigue resistance and surface roughness of acetal resin clasp and Co-Cr clasp after cycling for further work.

\section{Conclusion}

In accordance with limitations of this study, it could be concluded that:

a. Acetal resins clasps ( $1.0 \mathrm{~mm}$ in cross section diameter) engage $0.25 \mathrm{~mm}$ undercut provide sufficient retention nearly similar that of Co-Cr clasps.

b. The acetal resin clasps should be thicker more than $1.0 \mathrm{~mm}$ (in cross-sectional diameter) when engage a deeper undercut ( 0.50 $\mathrm{mm}$ ) to gain the stiffness of the cast Co-Cr clasps $1 \mathrm{~mm}$ in cross sectional diameter and to obtain clinically acceptable retention.

\section{References}

1. Helal MA, Baraka OA, Sanad ME, Ludwig K, Kern M (2012) Effects of long-term simulated RPD clasp attachment/detachment on retention loss and wear for two clasp types and three abutment material surfaces. J Prosthodont 21: 370-7.

2. Helal MA, Baraka OA, Sanad ME, Al-Khiary Y, Ludwig K, et al. (2014) Effect of clasp design on retention at different intervals using different abutment materials and in a simulated oral condition. J Prosthodont 23: 140-5.

3. Taleb FAA, Eltorky IR, El-Sheikh MM, Moula SA (2013) Patient Satisfaction and Radiographyical Evaluation of Acetal Resin Retentive Clasp Arm versus Conventional Clasp on Abutment Teeth in Upper Unilateral Removable Partial Dentures. J Amer Sci 9: 425-31.

4. Kotake M, Wakabayashi N, Ai M, Yoneyama T, Hamanaka H (1997) Fatigue resistance of titanium-nickel alloy cast clasps. Int J Prosthodont 10: 547-52.

5. Rodrigues RC, Ribeiro RF, de Mattos Mda G, Bezzon OL (2002) Comparative study of circumferential clasp retention force for titanium and cobalt-chromium removable partial dentures. J Prosthet Dent 88: 290-96.

6. Vallittu PK, Kokkonen M (1995) Deflection fatigue of cobalt-chromium, titanium, and gold alloy cast denture clasp. J Prosthet Dent 74: 412-19.

7. Wang RR, Fenton A (1996) Titanium for prosthodontic applications: a review of the literature. Quintessence Int 27: 401-08.

8. VandenBrink JP, Wolfaardt JF, Faulkner MG (1993) A comparison of various removable partial denture clasp materials and fabrication procedure for placing clasps on canine and premolar teeth. J Prosthet Dent 70: 180-8.

9. Firtell DN (1968) Effect of clasp design upon retention of removable partial dentures. J Prosthet Dent 20: 43-52.

10. Bates JF (1963) Retention of cobalt-chromium partial dentures. Dent Pract 14: 168-71.

11. Soo S, Leung T (1996) Hidden clasps versus C clasps and I bars: a comparison of retention. J Prosthet Dent 75: 622-5.

12. Bergman B, Hugoson A, Olsson CO (1995) A 25 year longitudinal study of patients treated with removable partial dentures. J Oral Rehabil 22: 595-9.

13. Mine K, Fueki K, Igarashi Y (2009) Microbiological risk for periodontitis of abutment teeth in patients with removable partial dentures. J Oral Rehabil 36: 696-702.

14. Tannous F, Steiner M, Shahin R, Kern M (2012) Retentive forces and fatigue resistance of thermoplastic resin clasps. Dent Mater $28: 273-8$.

15. Moreno de Delgado M, Garcia LT, Rudd K.D (1986) Camouflaging partial denture clasps. J Prosthet Dent 55: 656-60.

16. Brudvik JS, Palacios R (2007) Lingual retention and the elimination of the visible clasp arm. J Esthet Restor Dent 19: 247-54.

17. Chu CH, Chow TW (2003) Esthetic designs of removable partial dentures. Gen Dent 51: 322-4. 
18. Sykes LM, Dullabh HD, Sukha AK (2002) Use of technopolymer clasps in prostheses for patients due to have radiation therapy. SADJ 57: 29-32.

19. Fitton JS, Davies EH, Howlett JA, Pearson GJ (1994) The physical properties of a polyacetal denture resin. Clin Mater 17: 125-9.

20. Turner JW, Radford DR, Sherriff M (1999) Flexural properties and surface finishing of acetal resin denture clasps. J Prosthodont 8: 188-95.

21. Arda T, Arikan A (2005) An in vitro comparison of retentive force and deformation of acetal resin and cobalt-chromium clasps. J Prosthet Dent 94: 267-74.

22. Jean C W, George H L, Russell A W, Robert L S, Mark S (2003) In vitro deformation of acetal resin and metal alloy removable partial denture direct retainer. J Prosthetic Dent 90: 586-96.

23. Arikan A, Ozkan YK, Arda T, Akalin B (2005) An in vitro investigation of water sorption and solubility of two acetal denture base materials. Eur J Prosthodont Restor Dent 13: 119-22.

24. Ozkan Y, Arikan A, Akalin B, Arda T (205) A study to assess the colour stability of acetal resins subjected to thermocycling. Eur J Prosthodont Restor Dent 13: $10-4$.

25. Kim D, Park C, Yi Y, Cho L (20004). Comparison of cast Ti-Ni alloy clasp retention with conventional removable partial denture clasps. J Prosthet Dent 91: 374-82.

26. Kotake M, Wakabayashi N, Ai M, Yoneyama T, Hamanaka H (1997) Fatigue resistance of titanium-nickel alloy cast clasps. Int J Prosthodont 10: 547-52.

27. Kurtz SM, Devine JN (2007) PEEK biomaterials in trauma, orthopedic, and spinal implants. Biomaterials 28: $4845-69$.

28. Sato Y, Yuasa Y, Akagawa Y, Ohkawa S (1995) An investigation of preferable taper and thickness ratios for cast circumferential clasp arms using finite element analysis. Int J Prosthodont 8: 392-7.

29. Frank RP, Nicholls JI (1981) A study of the flexibility of wrought wire clasps. J Prosthet Dent 1981; 45:259-67.

30. Chu CH, Chow TW (2003) Esthetic designs of removable partial dentures. Gen Dent. 51: 322-4.

31. Meenakshi A, Gupta R, Bharti V, Sriramaprabu G, Prabhakar R (2016) An Evaluation of Retentive Ability and Deformation of Acetal Resin and CobaltChromium Clasps. JCDR 10: ZC37-41.

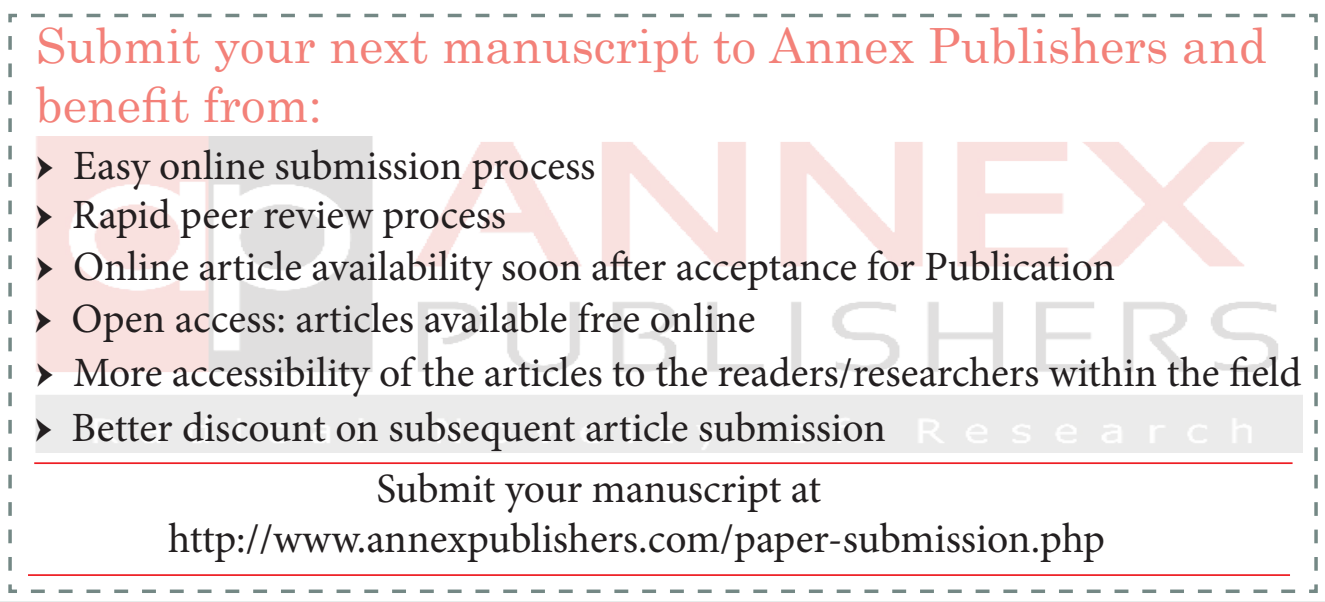

